\title{
Computer simulations of thermal phenomena in surface heating process using the real distribution of $\mathrm{Yb}$ :YAG laser power
}

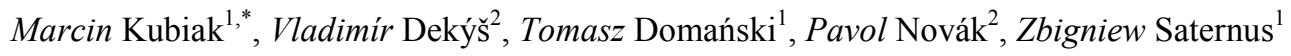 \\ ${ }^{1}$ Czestochowa University of Technology, Institute of Mechanics and Machine design Foundations, \\ Dabrowskiego 73, 42-200 Czestochowa, Poland \\ ${ }^{2}$ University of Zilina, Department of Applied Mechanics, Univerzitna 1, 01026 Zilina, Slovak rep.
}

\begin{abstract}
This work concerns mathematical and numerical modelling of temperature field during Yb:YAG laser heating of sheets made of S355 steel with the motion of liquid steel in the fusion zone taken into account. Laser power distribution and the caustics are determined on the basis of the geostatistical kriging method. Temperature field and melted material velocity field in the fusion zone are obtained from the numerical solution of continuum mechanics equations using projection method and finite volume method. Numerical algorithms are implemented into computer solver using ObjectPascal programming language. Computer simulations of Yb:YAG laser heating process are performed for different process parameters. Characteristic zones of experimentally obtained cross sections of heated elements are compared to numerically predicted fusion zone and heat affected zone.
\end{abstract}

Keywords: Yb:YAG laser, laser heating, heat affected zone, numerical modelling

\section{Introduction}

The heat treatment of metals is a process known for ages. Studied theoretically in terms of thermal, structural and mechanical phenomena [1-3]. Nowadays, diode pumped Yb:YAG lasers are intensively studied in terms of the use of new laser processing methods in the industry [4]. The study is performed in the field of experimental research as well as theoretical approach. The laser beam power and its distribution in heated element as well as liquid material motion in the fusion zone are major factors determining the temperature field in the laser beam processing.

Theoretical analysis of coupled physical phenomena accompanying laser-material interaction is very complex in terms of mathematical modelling and numerical solutions. Numerical models usually describe chosen phenomena, due to the computational complexity of the problem [5-8]. Consideration of liquid material motion in the model allows for the analysis of previously neglected phenomena in material melting processes and has a significant impact on the estimated temperature distribution $[6,9]$.

\footnotetext{
* Corresponding author: kubiak@imipkm.pcz.pl

Reviewers: Ladislav Écsi, Richard Pastirčák
} 
Moreover, changing focal length of focusing lens in the optical system determines the laser spot diameter as well as laser beam power intensity distribution [10,11]. Only a specific laser beam spot diameter is adopted in analytical models and the beam focusing as a technological parameter influencing the laser beam intensity distribution is neglected. Consequently, the laser beam intensity distribution models assumed in numerical analysis significantly differ from real Yb:YAG laser profile, obtained through experimental research.

A comprehensive theoretical model is presented in this study of thermal phenomena during Yb:YAG heating of S355 steel sheets, taking into account the real laser beam heat source intensity distribution and the motion of liquid steel in the fusion zone. In order to assess the quality of obtained results and comparison of predicted fusion zone and heat affected zone with experimental data is performed. Schematic sketch of analyses system is presented in Fig. 1.

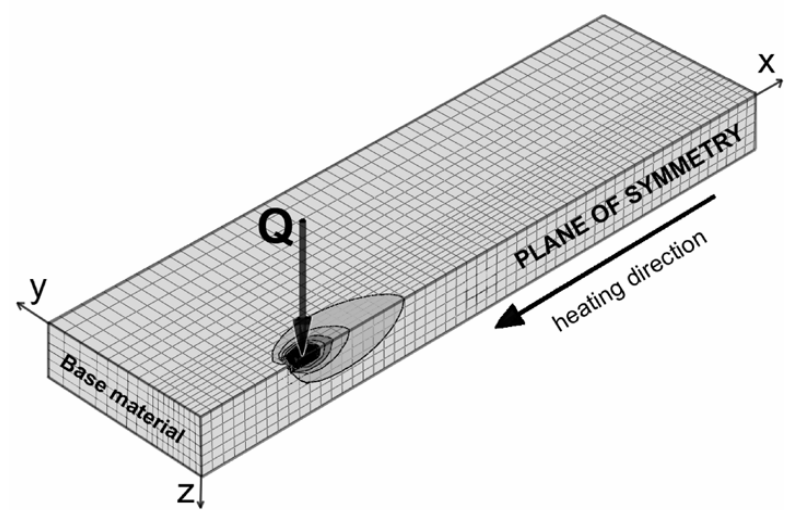

Fig. 1. Schematic sketch of laser heating simulation

\section{Numerical solutions}

The temperature field and liquid material velocity field are determined on the basis of the solution of mass, momentum and energy conservation equations. The motion of melted material in the fusion zone is mainly generated by natural convection according to Boussinesq model [12-15]. Mathematical model assumes fuzzy solidification front between solidus and liquidus temperatures where liquid material motion is treated as a fluid flow through porous medium with respect to Darcy's model. In theoretical considerations latent heat of fusion and evaporation are taken into account.

Initial conditions and boundary conditions complete governing equations. Momentum equation is completed by initial condition $t=0: \mathbf{v}=0$ and boundary conditions implemented at the welding pool boundary determined by solidus temperature $\left(T_{S}\right)$. Energy conservation equation is completed by initial condition: $t=0: T=T_{0}$ and boundary conditions, taking into account heat loss due to convection, radiation and evaporation.

Governing equations are numerically solved using projection method with finite volume method [16]. Interpolation algorithms are used for the description of heat source power distribution, allowing a precise description of $\mathrm{Yb}$ :YAG laser power intensity. Models elaborated on the basis of ordinary Kriging method [17] in the form of point Kriging take into account the real laser power distribution obtained in experimental research made on laser welding station equipped with TruDisk 12002 laser. Solution algorithm is presented in Fig. 2. 


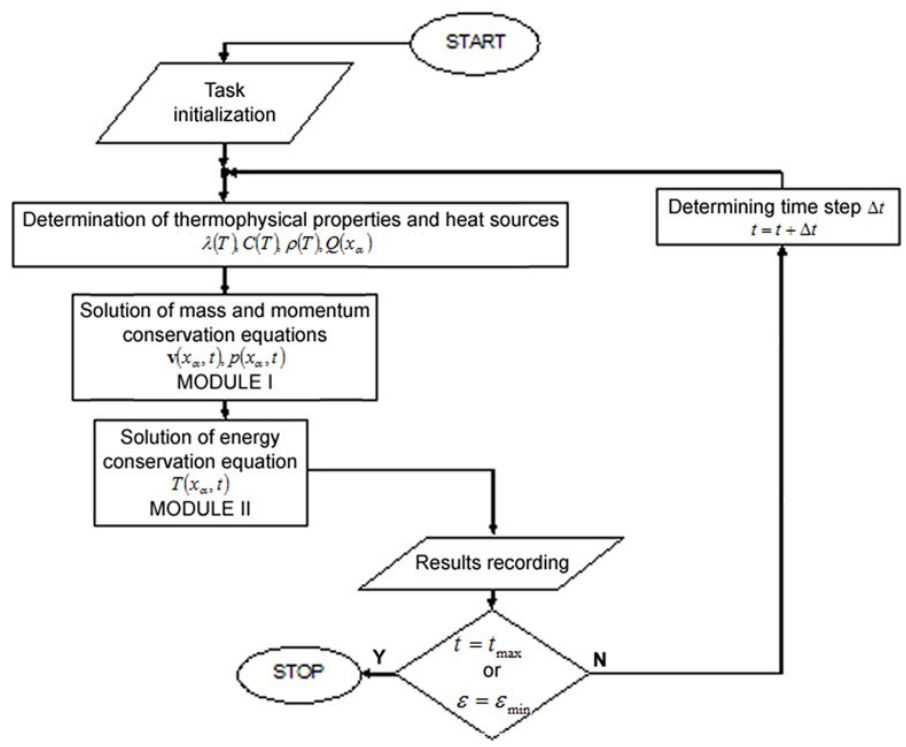

Fig. 2. Numerical solution algorithm

\section{Experimental research}

Trumpf D70 laser head combined with TruDisk 12002 disk laser is used in experimental research (Fig. 3a). The head is equipped with a collimator lens having a focal length $f_{c}=200 \mathrm{~mm}$ and a focusing lens having a focal length of $f=400 \mathrm{~mm}$. The fibre with a diameter $d L L K=400 \mu \mathrm{m}$ allows to obtain double magnification of the optical system, thus laser beam diameter is $d=0.8 \mathrm{~mm}$. Prometec UFF100 laser beam analysis system (Fig. 3b) is used in order to analyse the density of laser power distribution and the caustic of the laser beam.
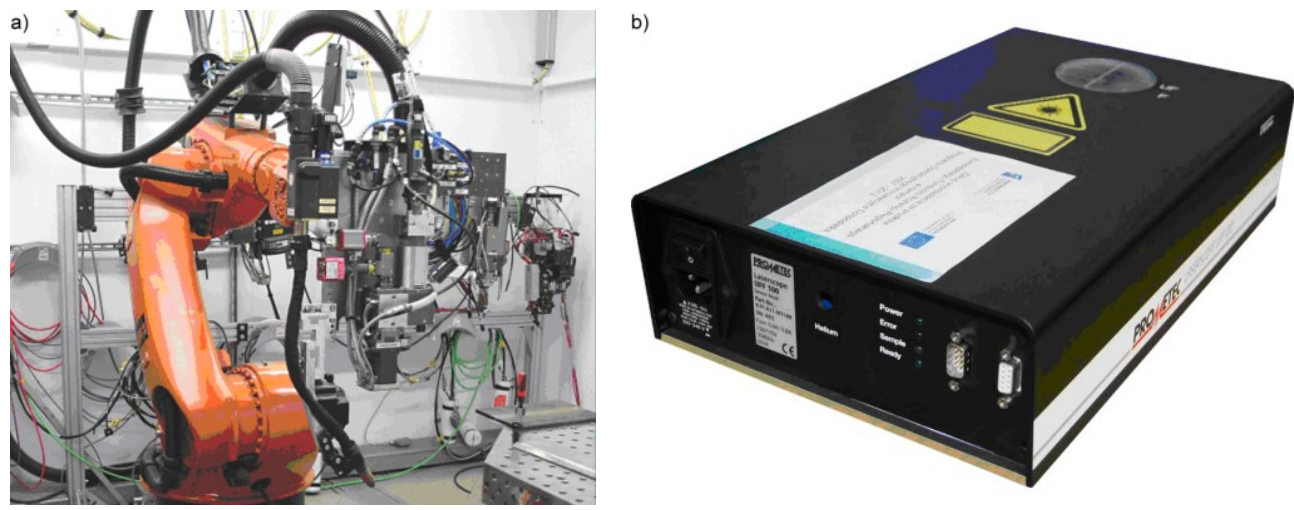

Fig. 3. Robotic laser welding station with a laser disk TruDisk 12002: a) D70 laser head mounted on the wrist of KUKA industrial robot and b) laser beam analyser UFF100

The laser heating experiment is made for S355 steel sheets with dimensions $250 \mathrm{~mm}$ in length, $50 \mathrm{~mm}$ in width, with a thickness of $5 \mathrm{~mm}$. Three heating processes are performed (Fig. 4) assuming laser head movement speed $v=3 \mathrm{~m} / \mathrm{min}$, the focusing point on the top surface of heated element $(z=0)$ and three different laser powers: $Q=900,500$ and $320 \mathrm{~W}$. 


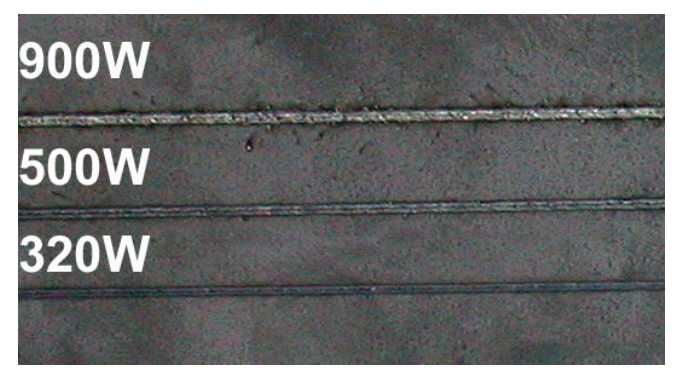

Fig. 4. Top surface of heated element for three different laser powers

\section{Results and discussion}

Numerical algorithms are implemented into computer solver using ObjectPascal programming language. Computer simulations of the $\mathrm{Yb}$ :YAG laser heating process are performed for S355 steel sheets with dimensions used in the experiment. The analysed domain is discretized by a staggered grid with the spatial step set to $0.1 \mathrm{~mm}$. The numerical analysis is made using interpolated heat source model and technological parameters assumed in experimental research. Basic parameters of the laser beam used in the model are obtained using Prometec UFF100 beam analyser, a diagnostic device for measuring high power lasers profile. Fig. 5 shows heat source power distribution obtained by Kriging method for interpolation grid step $\Delta h=0.02 \mathrm{~mm}$.
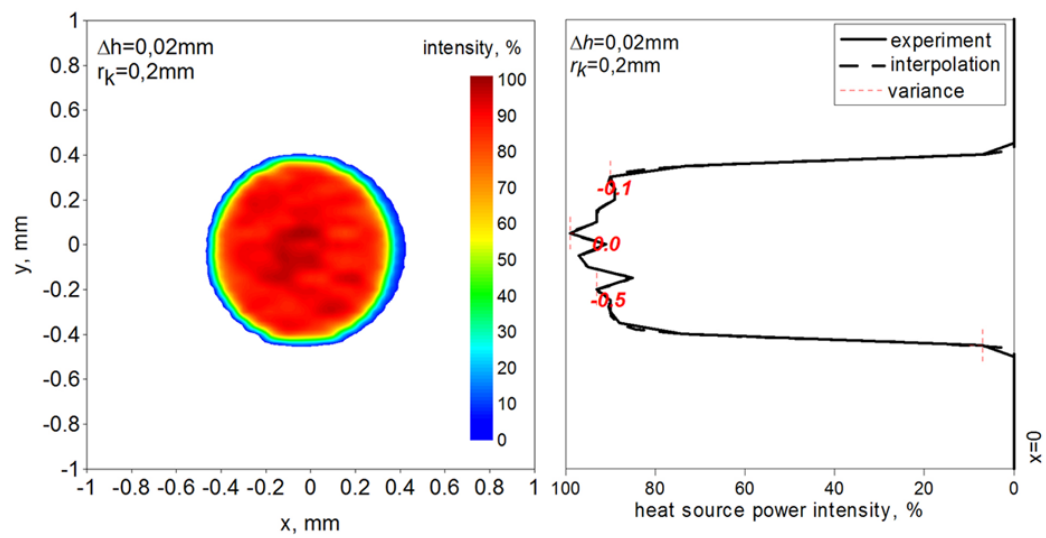

Fig. 5. Percentage distribution of laser beam power for $z=0$

Figure 6 illustrates the temperature distribution at the top surface and in cross section (for $x=3 \mathrm{~mm}$ ) of laser heated sheet. In Figure $6 \mathrm{~b}$ a comparison of predicted characteristic zones of heated element with the macroscopic picture of the cross section of heated element is presented. Solid line points out melted zone boundary (solidus temperature) whereas dashed line marks heat affected zone boundary (austenitization temperature, about 1000K). Figure 7 presents temperature distribution in the cross section of heated element maid for three different laser head powers in comparison with the macroscopic picture of the cross section of heated elements. 

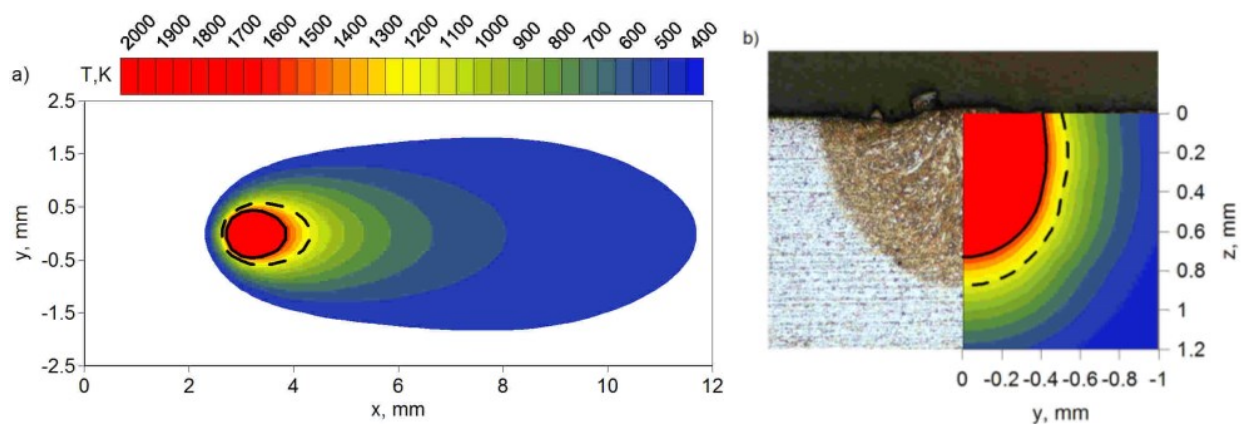

Fig. 6. Temperature distribution a) at the top surface and $\mathrm{b}$ ) in cross section (for $x=3 \mathrm{~mm}$ ) of laser heated sheet in comparison with macroscopic picture of the real heated element

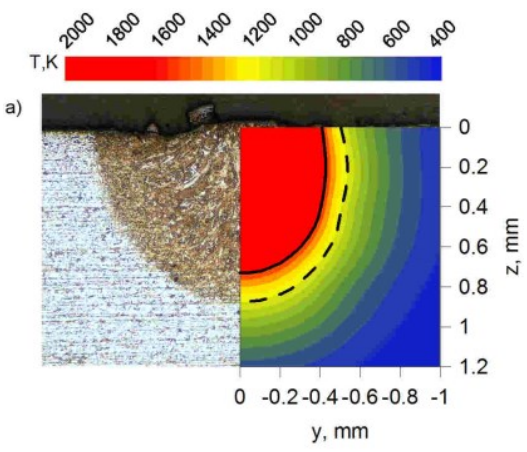

b)

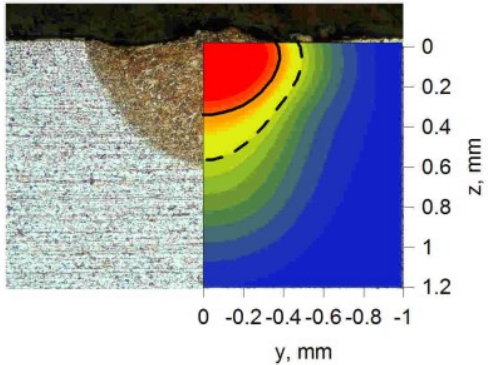

c)

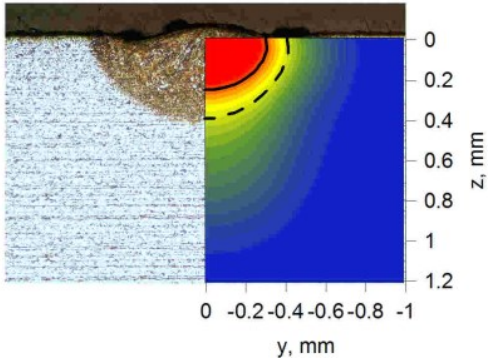

Fig. 7. Temperature distribution in the cross section (for $x=3 \mathrm{~mm}$ ) of laser heated sheets for three laser beam powers: a) $900 \mathrm{~W}$, b) $500 \mathrm{~W}$ and c) $320 \mathrm{~W}$ in comparison with macroscopic picture of the real heated elements 


\section{Conclusion}

Heat affected zone in laser heated elements is narrow, up to about $1.2 \mathrm{~mm}$ in width for $900 \mathrm{~W}$ laser beam power decreasing to about 0.8 for laser beam power $320 \mathrm{~W}$. Melted zone that appear for each experiment is also decreasing with decreasing laser beam power. Predictably, the use of lower laser beam power contributed to the reduction of melted area and heat affected zone.

Application of Kriging algorithm allowed to use experimental studies in numerical analysis and for a precise reproduction of the real thermal load. Performed comparison of the heat source power distribution model with experimental data as well as characteristic zones of heated elements corroborates the validity of performed theoretical assumptions and usability of model in practical applications.

\section{References}

1. S. H. Kang, Y. T. Im, Three-dimensional thermo-elasto-plastic finite element modeling of quenching process of plain carbon steel in coulee with phase transformation. J. Mater Proc Technol. 192-193, 381-390 (2007)

2. A. Bokota, T. Domanski, Modelling and numerical analysis of hardening phenomena of tools steel elements. Arch Metall Mater. 54 (3), 575-587 (2009)

3. T. Domanski, A. Sapietova, M. Saga, Application of Abaqus software for the modeling of surface progressive hardening. Procedia Engineering 177, 64-69 (2017)

4. D. Richardson, J. Nilsson, W. Clarkson, High power fiber lasers: current status and future perspectives. J. Opt Soc Am. B.27, 63-92 (2010)

5. A. De, T. Debroy, Reliable calculations of heat and fluid flow during conduction mode laser welding through optimization of uncertain parameters. Welding Journal 84101 $112(2005)$

6. X. Jin, L. Li, Y. Zhang, A heat transfer model for deep penetration laser welding based on an actual keyhole. Int J Heat Mass Tran. 46, 15-22 (2003)

7. L. Han, F.W. Liou, Numerical investigation of the influence of laser beam mode on melt pool. Int J Heat Mass Tran. 47, 4385-4402 (2004)

8. J. Winczek, A. Modrzycka, E. Gawronska, Analytical description of the temperature field induced by laser heat source with any trajectory. Procedia Engineering 149, 553-558 (2016)

9. M. Kubiak, W. Piekarska, Comprehensive model of thermal phenomena and phase transformations in laser welding process. Computers \& Structures 172, 29-39 (2016)

10. M. Kubiak, W. Piekarska, S. Stano, Modelling of laser beam heat source based on experimental research of Yb: YAG laser power distribution, Int J Heat Mass Tran, 83, 679-689 (2015)

11. M. Kubiak, W. Piekarska, Z. Saternus, T. Domański, Numerical prediction of fusion zone and heat affected zone in hybrid $Y b$ : YAG laser $+G M A W$ welding process with experimental verification. Procedia Engineering 136, 88-94 (2016)

12. R. Gnatowska, T. Rybak, Numerical Analysis of Heat Transfer around 2D Circular Cylinder in Pulsation Inflow. AIP Conference Proceedings 1648, 850125 (2015)

13. R. Dyja, E. Gawronska, A. Grosser, Numerical problems related to solving the NavierStokes equations in connection with the heat transfer with the use of FEM. Procedia Engineering 177, 78-85 (2016) 
14. T. Skrzypczak, E. Węgrzyn-Skrzypczak, Modeling of thermal contact through gap with the use of finite element method. J Appl Math. Comput Mech. 14 (4), 145-152 (2015)

15. T. Skrzypczak, A finite element multi-mesh approach for heat transport between disconnected regions. Procedia Engineering 177, 204-209 (2017)

16. S. V. Patankar, Numerical heat transfer and fluid flow. (Taylor \& Francis, USA, 1990)

17. M. A. Oliver, R. Webster, Kriging: a method of interpolation for geographical information system. Int. J Geogr Inf Sys. 4, 313-332 (1990) 